\title{
Karyological Studies on Some Bird Species
}

\author{
Ahmed E. Yaseen \\ Cytogenetic Lab. Zoology Dept. Faculty of Science (Qena), South Valley University, Egypt
}

Accepted January 26, 1998

\begin{abstract}
Summary The chromosome complements of eight species of Egyptian birds are investigated. The diploid chromosome numbers of these species, Hirundo daurica, Turdus migratorius, Corvus splendens, Bubulcus ibis, Ardeola grayii, Meleagris gallopavo, Upupa epops and Columba oenas, 74, 58, $78,60,60,58,110$ and 76 are respectively. The sex chromosome constitutions are of homogametic $\mathrm{ZZ}$ in males and of heterogametic $\mathrm{ZW}$ in females. Karyotypic and morphometric data for the chromosome complements of these species have also been studied.
\end{abstract}

In the framework of the available cytogenetic techniques, avian karyological studies have always been areas of those mammals. This is mainly because of inherent difficulties in handling avian chromosomes. In the class Aves which comprises more than 8500 species, only about $6-7 \%$ of them have been karyologically investigated as compared to $30 \%$ of mammalia (Tegelstrom et al. 1983, De Boer 1984, Sultana and Bhunya 1987, Sasaki et al. 1994).

Bird karyotypes are characterized by the presence of many small individually indistinguishable microchromosomes in addition to larger macrochromosomes. Large microchromosomes may fuse by Robertonian translocation to create small metacentric chromosomes. Bird orders representing $65 \%$ of all species, that were investigated, have a diploid chromosome number of 78 to 82 with 7 to 9 pairs of macrochromosomes. Most remaining species have a karyotype with a lower diploid number of chromosomes of 66 to 74 showing 12 pairs of macrochromosomes and 21 to 25 pairs of microchromosome (Carlenius et al. 1981, Tegelstrom et al. 1983).

Cytogenetical studies of different species of birds from various families were previously carried out by different workers (Ray-Chaudhuri 1969, 1973, Hamnar 1970, Benrischke and Hsu 1971, Teglstran and Ryttman 1981, De Boer 1984).

Although there have been considerable development in the field of cytotaxonomic studies in the last twenty years, yet very little information are available on the chromosomal characters of birds as a whole. In order to fill this lacuna, the aim of the present work is to describe in detail the karyotypes of these eight species (Hirundo daurica, Turdus migratorius, Corvus splendens, Bubulcus ibis, Ardeola grayii, Meleagris gallopavo, Upupa epops and Columba oenas), in order to contribute to the knowledge of the avian cytogenetics.

\section{Materials and methods}

Source of animals: Individuals of eight species, Hirundo daurica ( 3 males and 2 females), Turdus migratorius (2 males and 4 females), Corvus splendens ( 3 males and 3 females), Bubulcus ibis (4 males and 3 females), Ardeola grayii (3 males and 2 females), Meleagris gallopavo (2 males and 2 females), Upupa epops ( 2 males and 1 female) and Columba oenas ( 2 males and 2 females), were purchased from animal dealer from different localities around Qena City (Farshout, Deshna, Qift and Quse) in Upper Egypt. The identification and classification of the specimens was carried out by the help of the descriptions and keys provided by Gianfranco (1984) and Steven et al. (1989).

Chromosomal preparation: Chromosomal analysis was carried out on the bone marrow cells 
by the air drying method of Adegoke and Nadesan (1986) with some modification made by Bhunya and Das (1991) and Salama et al. (1995) as follows: Individuals were injected intraperitonally with $0.6 \mathrm{mg} / \mathrm{kg}$ solution of colchicine and sacrificed after $2 \mathrm{hr}$. Both femurs were immediately removed and femoral contents were flushed with phosphate saline into small tube by using a small syringe needle (26 gauge). The cell suspension was centrifuged at $1000 \mathrm{rpm}$ for $5 \mathrm{~min}$.

A hypotonic solution $(0.075 \mathrm{M} \mathrm{KCI})$ was added to cell pellet and let stand for $12 \mathrm{~min}$ at $37^{\circ} \mathrm{C}$, then centrifuged. The cells were then fixed in Carnoy's solution for $10 \mathrm{~min}$ and resuspended two or three times in fresh fixative to ensure fixation. Slides were prepared by dropping three or four drops of cell suspension on pre-cleaned glass slides. The slides were then stained for $5 \mathrm{~min}$ in a warm $10 \%$ Giemsa solution. Preparations were observed and photographed on a Leitz Dialux microscope. Chromosomes were arranged according to size terminology for centromeric position follow Levan et al. (1964). The fundamental number (FN) for each species was calculated on the basis that, metacentrics, submetacentrics and subtelocentrics have a value of two while telocentric has a value of one (except the sex chromosomes).

(a)

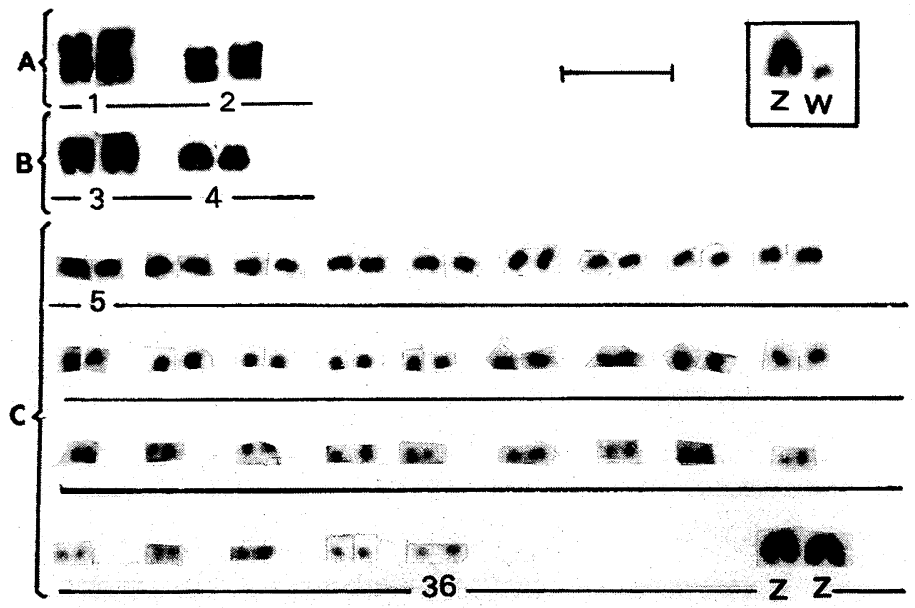

(b)

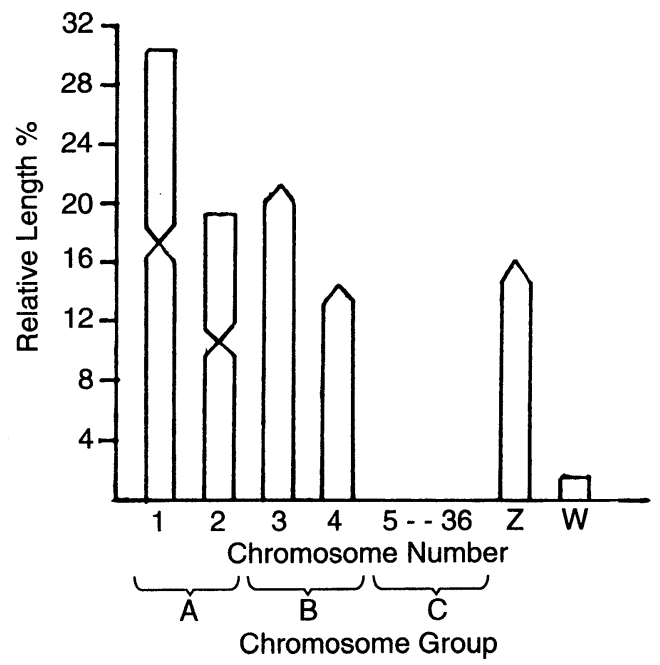

Fig. 1. Male karyotype (a) and female idiogram (b) of Hirunda daurica; insert are the sex chromosomes from a female. Bar $=10 \mu \mathrm{m}$. 
(a)

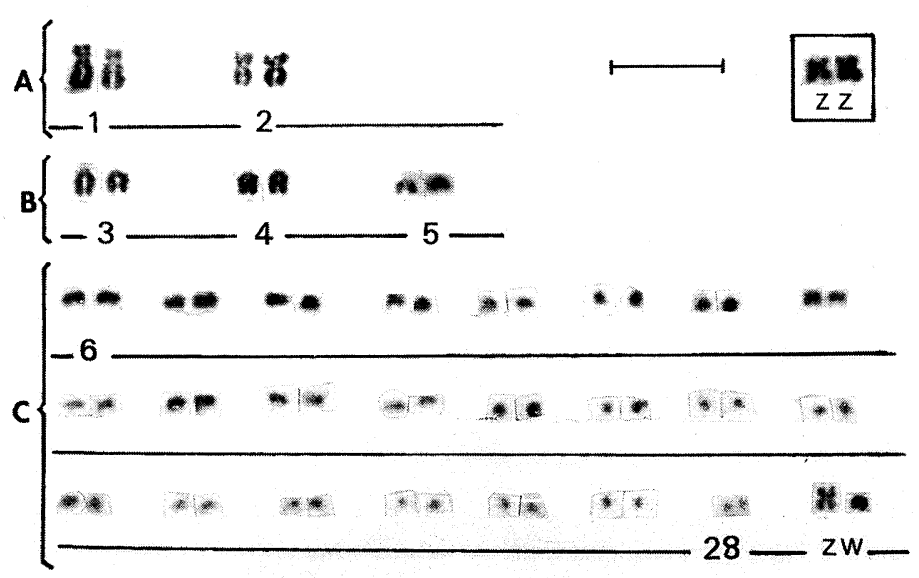

(b)

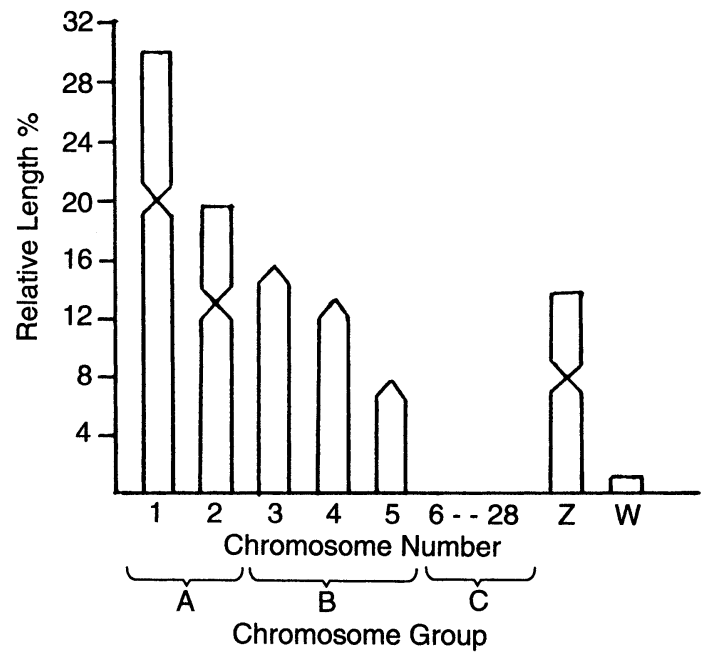

Fig. 2. Karyotype (a) and idiogram (b) of female Turdus migratorius; insert are the sex chromosomes from a male. $\mathrm{Bar}=10 \mu \mathrm{m}$.

\section{Results and discussion}

In the karyotype, chromosomes with definite centromeric position and those that can be measured with some degree of confidence have been considered as macrochromosomes and the rest of them are microchromosomes. For all the species examined, at least 20 metaphase spreads were scored from bone marrow preparations in each sex.

\section{Order: Passeriforms}

a) Hirundo daurica: The diploid chromosome number has been determined to be $(2 n=74$, $\mathrm{FN}=12$ ), ten of them were macrochromosomes including the $\mathrm{ZZ}$ chromosomes, while the rest, 64, were microchromosomes. The macrochromosomes were divided into two groups (Fig. 1a): Group A consists of two pairs of metacentrics and group B consists of two pairs of acrocentric chromosomes. The sex chromosomes were identified in the karyotype: the Z-chromosome is acrocentric while the W-chromosome is a microchromosome. The results of this species are considered to be new in the current lest of Bird karyotype. 
(a)

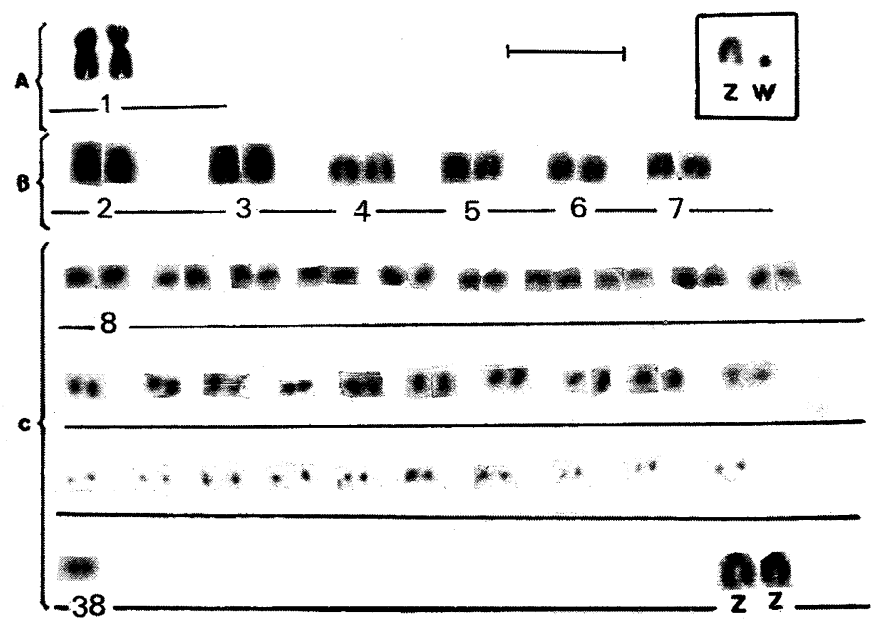

(b)

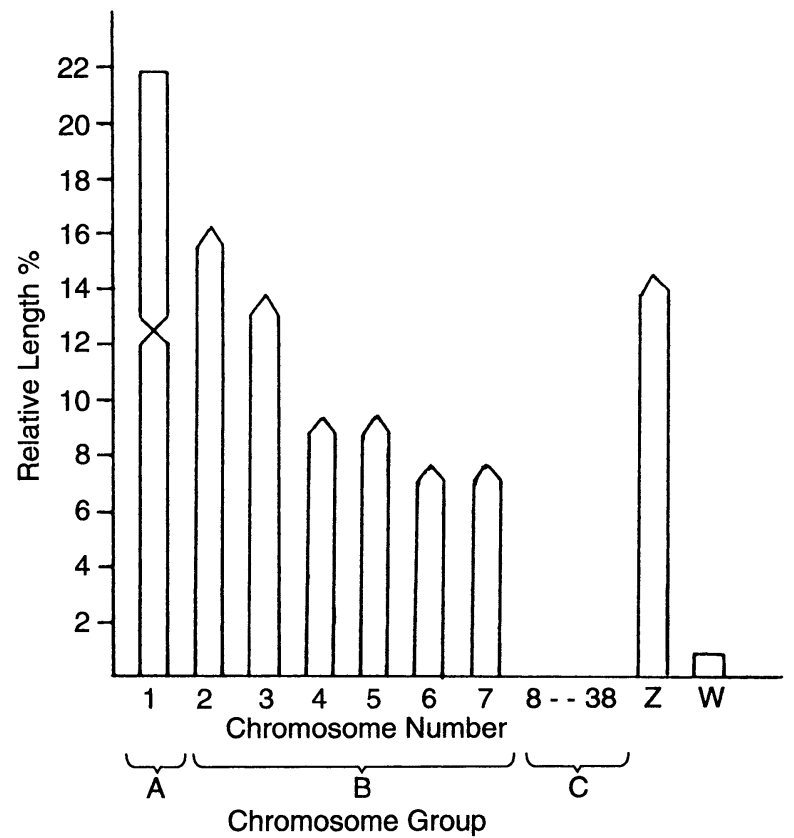

Fig. 3. Male karyotype (a) and female idiogram (b) of Corvus splendens; insert are the sex chromosomes from a female. $\mathrm{Bar}=10 \mu \mathrm{m}$.

b) Turdus migratorius: The karyotype of this species contained $12(10+\mathrm{ZZ})$ macrochromosomes and 46 microchromosomes with $\mathrm{FN}=14$. The macrochromosomes were divided into two groups (Fig. 2a): Group A has two pairs of submetacentric while group B has three pairs of acrocentric chromosomes. In the female karyotype $\mathrm{Z}$ and $\mathrm{W}$-chromosomes were identified: the Z-chromosome is metacentric while the $\mathrm{W}$-chromosome is microchromosome.

c) Corvus splendens: The diploid chromosome number ascertained for this species was 78 and $\mathrm{FN}=16$. The macrochromosomes were divided into two groups (Fig. 3a): Group A has one pair of metacentrics while group B has 6 pairs of acrocentric chromosomes. The Z-chromosome was acrocentric while the $\mathrm{W}$-chromosome could be one of microchromosomes.

Tables $1-3$ and Figs. $1 \mathrm{~b}, 2 \mathrm{~b}$ and $3 \mathrm{~b}$ are appended the morphometric and other details of various chromosomes in the complement and the idiogram on the basis of individual chromosome length in 
(a)

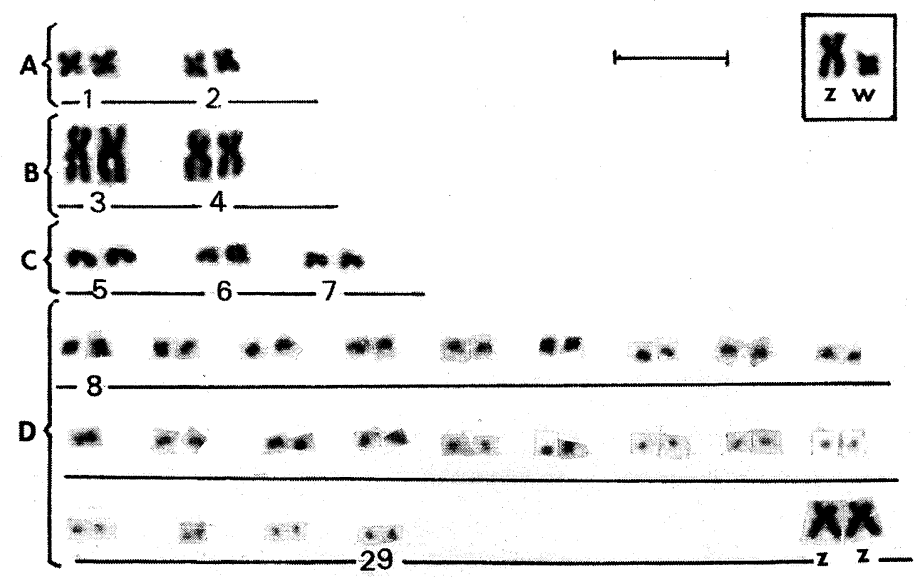

(b)

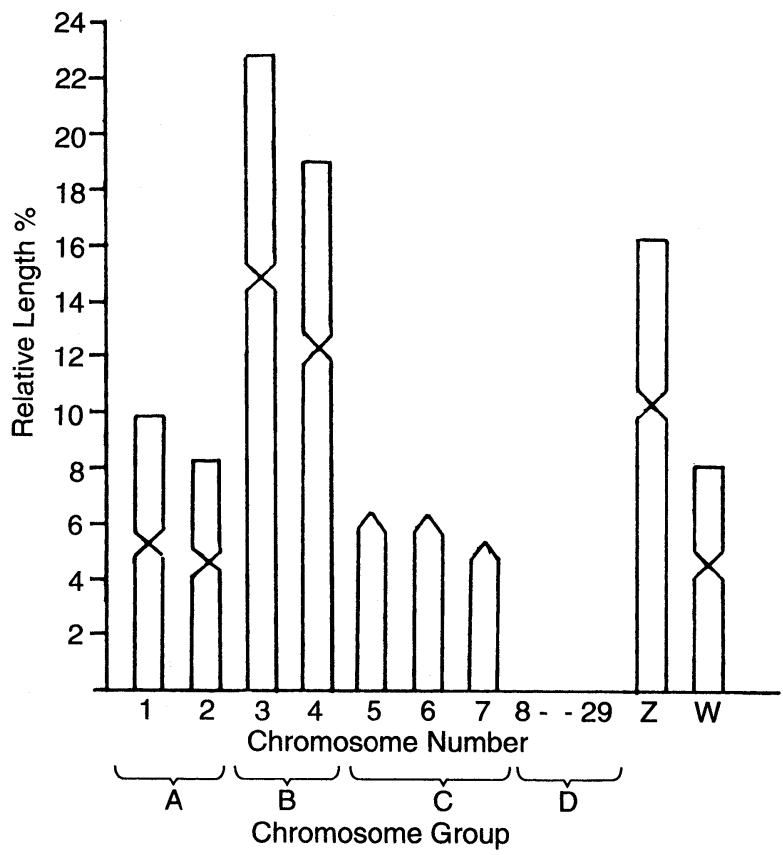

Fig. 4. Male karyotype (a) and female idiogram (b) of Bubulcus ibis; insert are the sex chromosomes from a female. Bar $=10 \mu \mathrm{m}$.

the three species, Hirundo daurica, Turdus migratorius and Corvus splendens, respectively.

\section{Order: Ciconiformes}

a) Bubulcus ibis: The diploid chromosome number assigned to this species was 60 with $\mathrm{FN}=22$ as revealed in the majority of metaphase plates (Fig. 4a). The macrochromosomes in the karyotype consisted of three group: Group A has two pairs of metacentrics, group B has two submetacentric pairs and group $\mathrm{C}$ has three pairs of acrocentric chromosomes. In the female karyotype the $\mathrm{Z}$-chromosome is submetacentric and the $\mathrm{W}$-chromosome is a metacentric one.

b) Ardeola grayii: In the karyotype of this species $8(7+\mathrm{ZZ})$ macrochromosomes and $22 \mathrm{mi}-$ crochromosomes with $\mathrm{FN}=24$ could be recognized (Fig. 5a). The macrochromosomes were divided into three groups: Group A consists of three metacentric pairs, group B has two submetacentric 
(a)

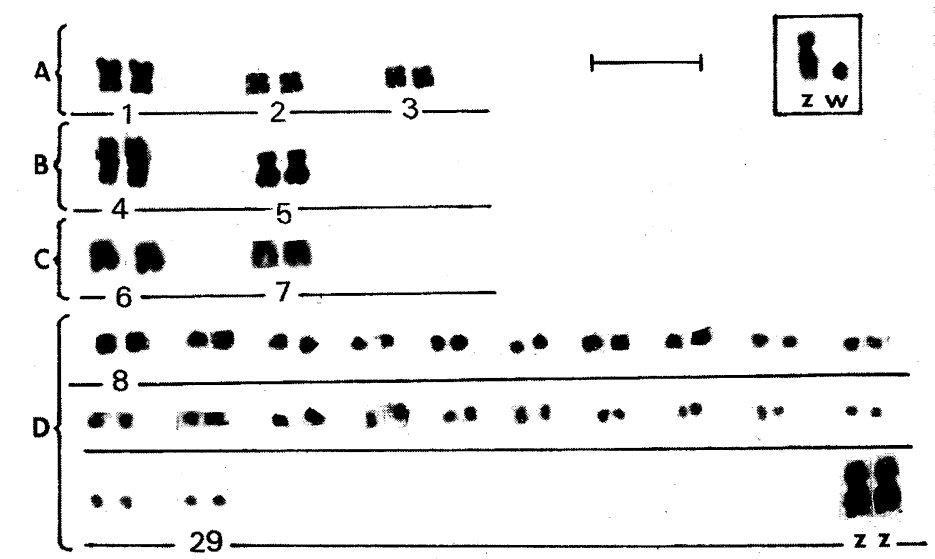

(b)

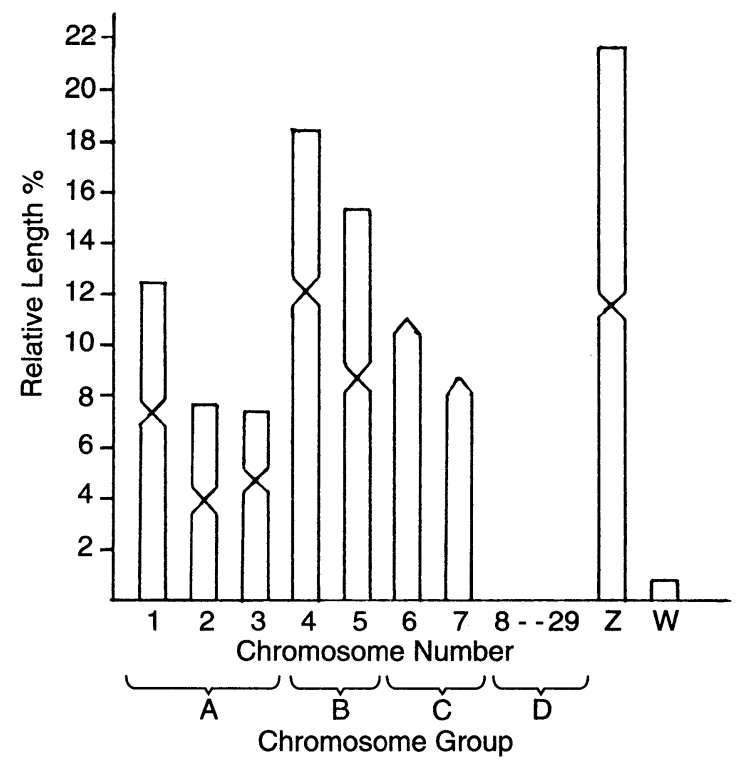

Fig. 5. Male karyotype (a) and female idiogram (b) of Ardeda grayii; insert are the sex chromosomes from a female. $\mathrm{Bar}=10 \mu \mathrm{m}$.

pairs and group $\mathrm{C}$ has two pairs of acrocentric chromosomes. The Z-chromosome is metacentric while the $\mathrm{W}$-chromosome is a microchromosome.

Tables 4, 5 and Figs. 4b, 5b embody the morphological details of the various chromosomes in the complement and the idiograms showing the mean length of the chromosomes for the two species, Bubulcus ibis and Ardeola grayii, respectively.

\section{Order: Galliformes}

Meleagris gallopavo: The diploid number of 58 chromosomes with $\mathrm{FN}=16$ has been ascertained for this species (Fig. 6a). The karyotype revealed clear distinction between macro and microchromosomes. These are $7(6+\mathrm{ZZ})$ macrochromosomes and 22 microchromosomes. Of 6 macrochromosome pairs, one pair was metacentric, one pair was submetacentric and 4 pairs were acrocentric chromosomes. The Z-chromosome was acrocentric and the $\mathrm{W}$-chromosome was a microchromosome. The morphometric measurements and idiogram showing the relative length of the 
(a)

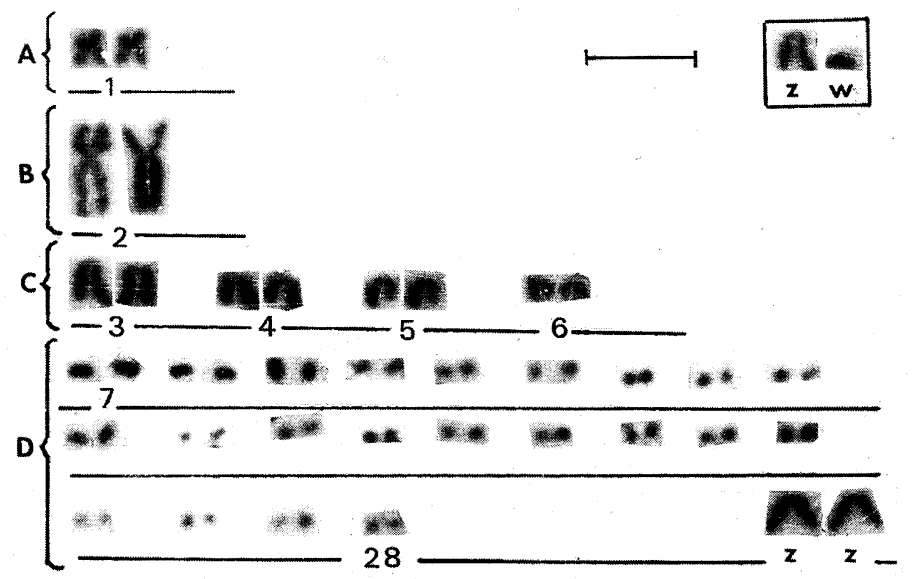

(b)

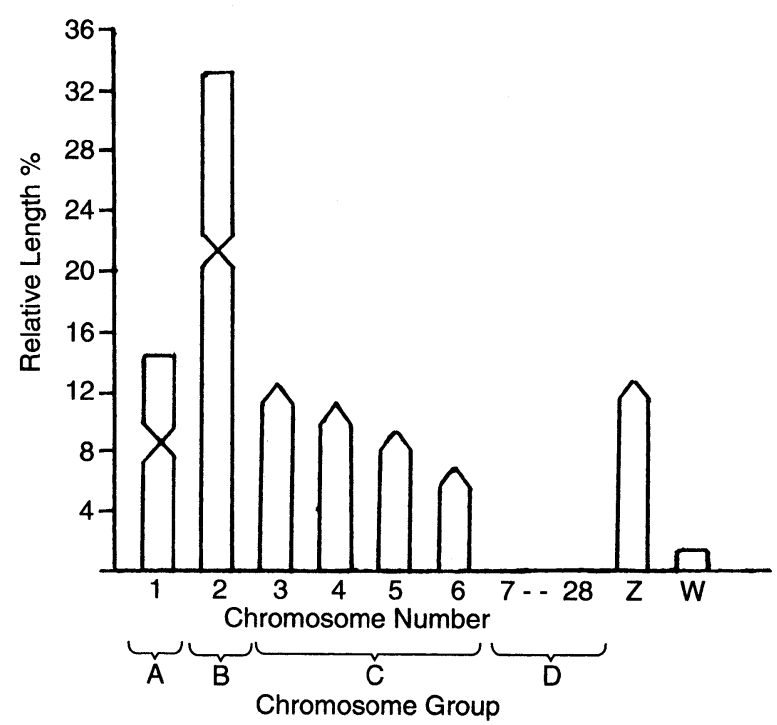

Fig. 6. Male karyotype (a) and female idiogram (b) of Meleagris gallopavo insert are the sex chromosomes from a female. Bar $=10 \mu \mathrm{m}$.

chromosomes are given in Table 6 and Fig. 6 a.

\section{Order: Coraciformes}

Upupa epops: In this species $28(26+\mathrm{ZZ})$ chromosomes were considered to be macrochromosomes (Fig. 7a). The karyotype consisted of four groups: Group A has two pairs of metacentrics, group $\mathrm{B}$ has four pairs of submetacentrics, group $\mathrm{C}$ has seven pairs of acrocentrics and group D has 41 microchromosomes. The sex chromosome were identified in the karyotype; the Z-chromosome is metacentric and the $\mathrm{W}$-chromosome is acrocentric. Table 7 summarize the morphometric data of macrochromosomes and the ideogram on the basis of individual chromosome length was given Fig. $7 b$.

\section{Order: Columbiformes}

Columba oenas: Chromosomal analysis for this species revealed that the diploid chromosome number is 76 with $\mathrm{FN}=30$ (Fig. 8a). The karyotype consisted of four groups: Group A has two pairs of metacentrics, group B has two pairs of submetacentrics, groups $\mathrm{C}$ has seven pairs of acro- 

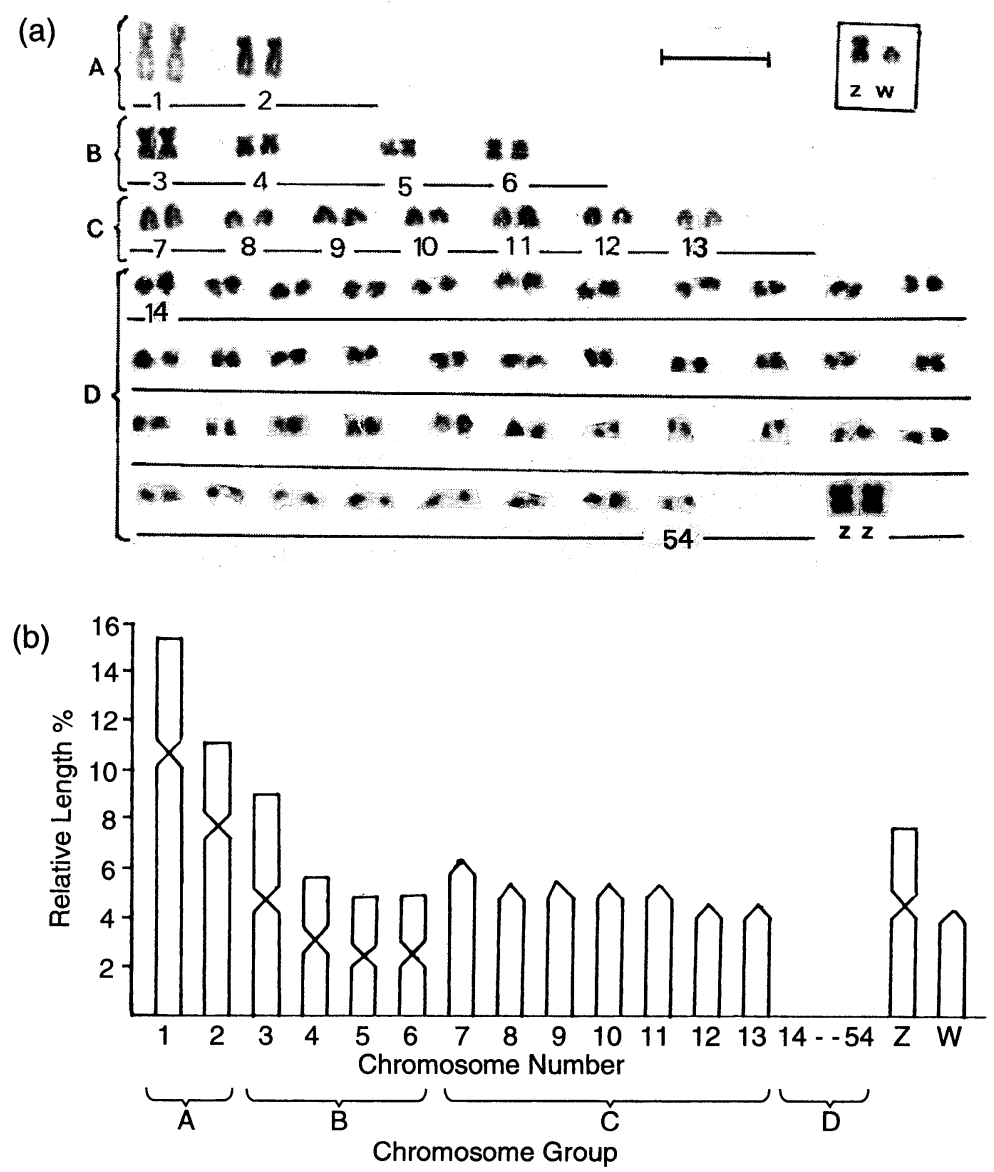

Fig. 7. Male karyotype (a) and female idiogram (b) of Upupa epops; insert are the sex chromosome from a female. Bar $=10 \mu \mathrm{m}$.

centrics and groups D has 26 mcirochromosomes. The sex chromosomes were identified: the Zchromosome is metacentric and W-chromosome is acrocentric. The results of this species are considered to be new in the current list of bird karyotype. Table 8 and Fig. $8 \mathrm{~b}$ revealed the chromosomal measurements and the ideogram of this species.

Karyology has been successfully applied to systematics of many groups of organisms as a differentiating tool for characterization of species and intraspecific groups. Unfortunately our knowledge of the structure of chromosome sets birds is still limited. The available data cannot provide enough basis for generalization on phylogenetic relationships of species within different group of birds.

In the present work, the basic diploid chromosome numbers for the species are clearly different except the two species Bubulcus ibis and Ardeola grayii. Despite these two species have the same diploid chromosome number of $2 n=30$, they are different in their chromosome, morphology.

Our results are in agreement with Tegelstrom and Ryttman (1981) who reported that the diploid chromosome numbers in birds (except Flaconiformes) range from 40 to 126 with a great tendency for an increase or decrease in the number of microchromosomes or macrochromosomes during the long-term evolution.

Y-chromosome of mammals and $\mathrm{W}$-chromosme of birds have several common features. They are generally small but gene(s) in both chromosomes are considered to play positive roles in trig- 
(a)

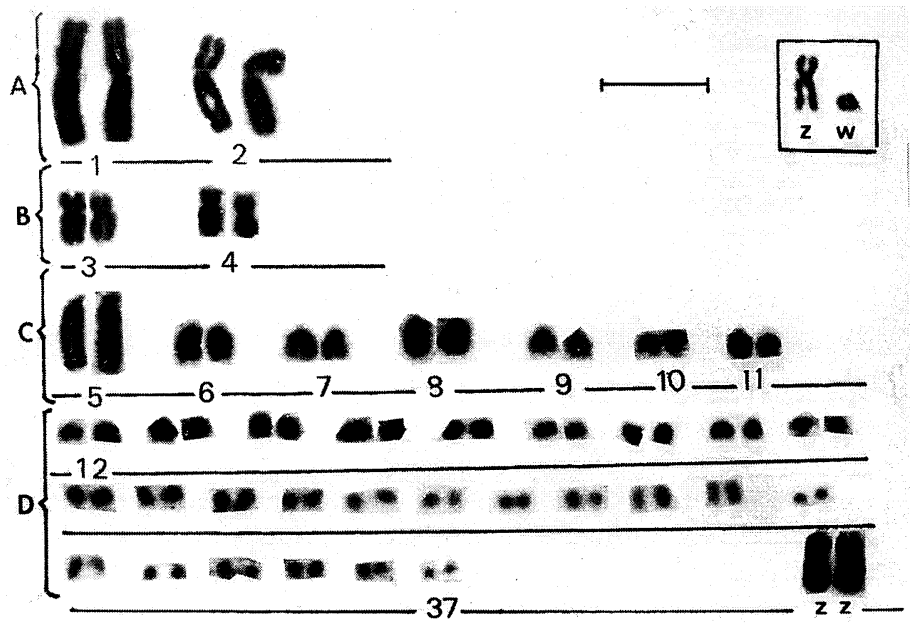

(b)

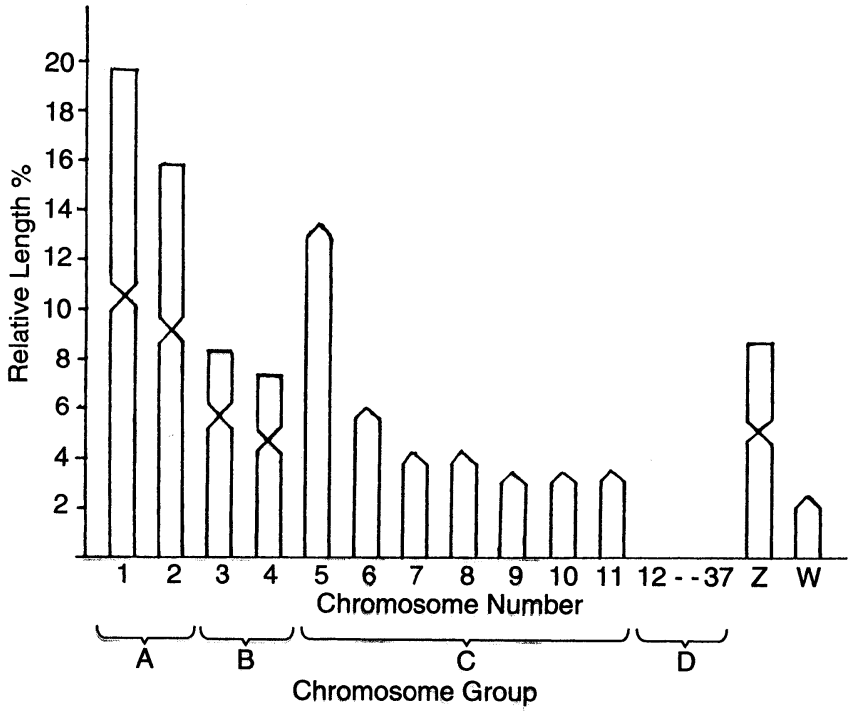

Fig. 8. Male karyotype (a) and female idiogram (b) of Columba oenas; insert are the sex chromosomes from a female. Bar $=10 \mu \mathrm{m}$.

gering testis determining and ovary determining processes, respectively (Mclaren 1988). Our results concerning the sex chromosomes for all species are in agreement with Mizuno (1991) who reported that the sex of most birds is female heterogametic; i.e. their sex chromosomes are $\mathrm{ZZ}$ of the male and $\mathrm{ZW}$ for the female. Thus an Ovum determines the genetic sex of a bird.

In conclusion, the study of the chromosomes in various species from comparative viewpoint is very significant and highly desirable from the viewpoint of taxonomy. The taxonomist of the future may build species definition as a fundamental ground composed of chromosome studies. 


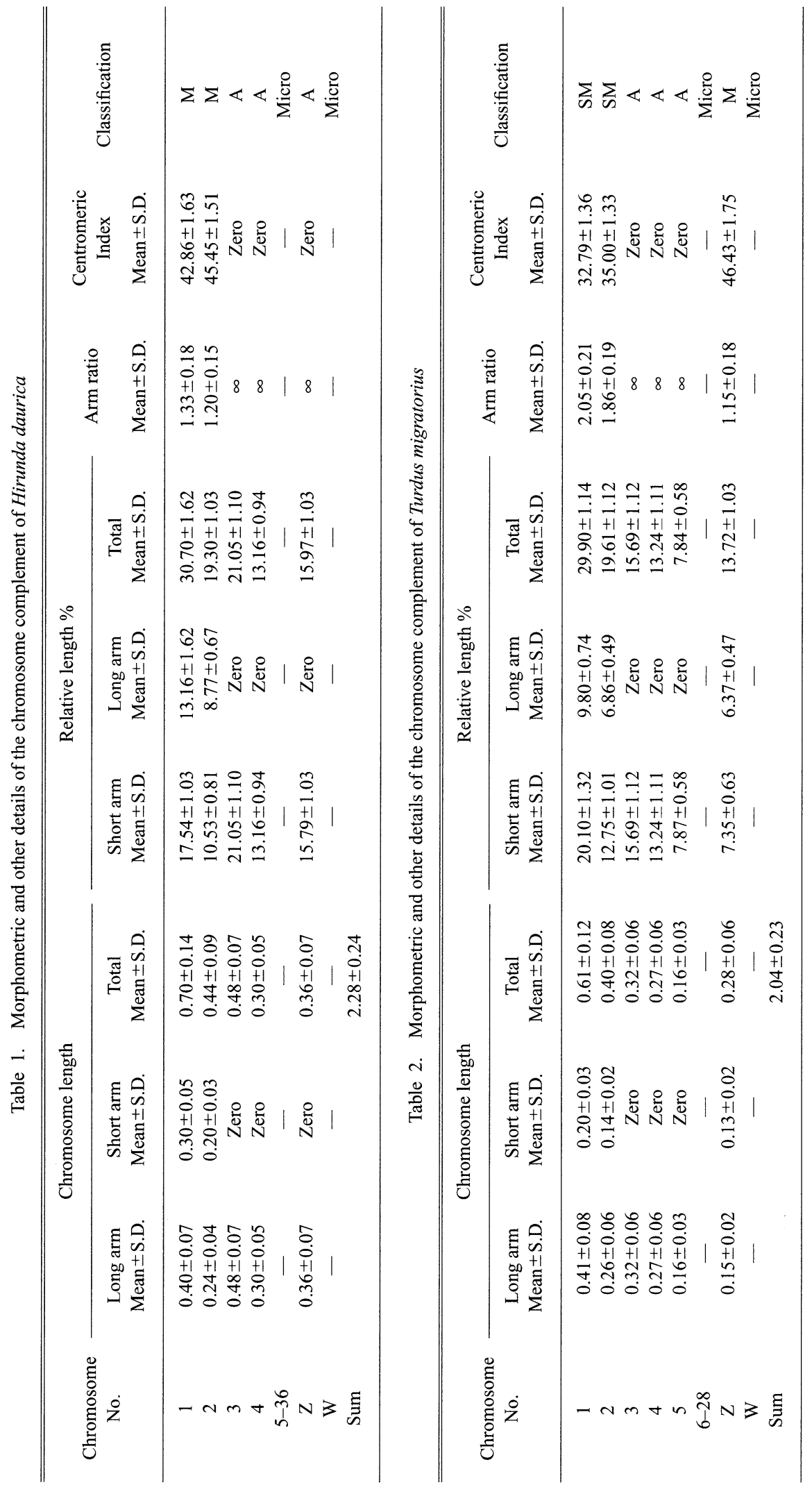



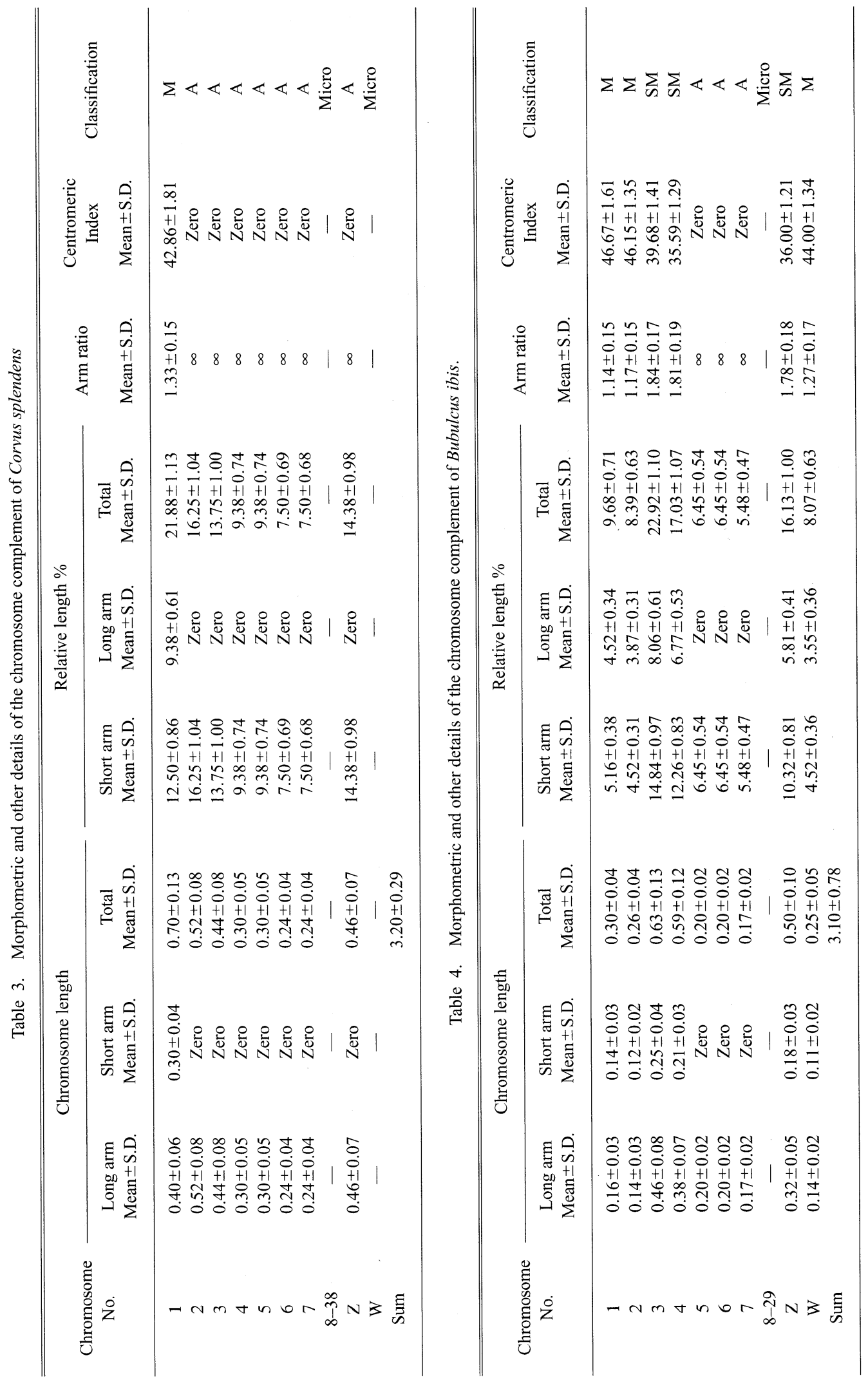


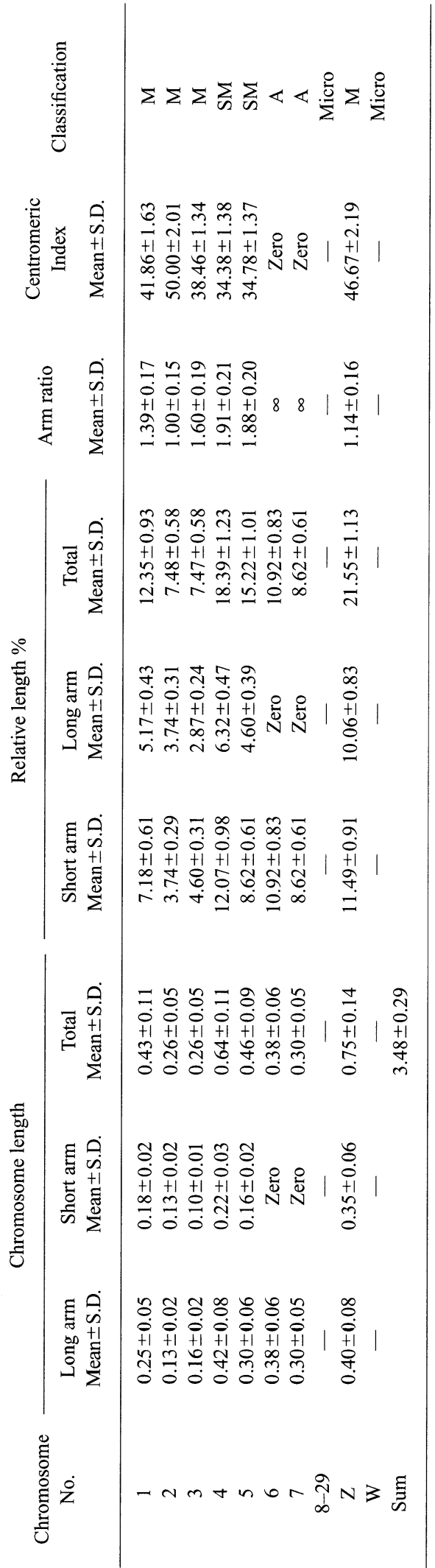

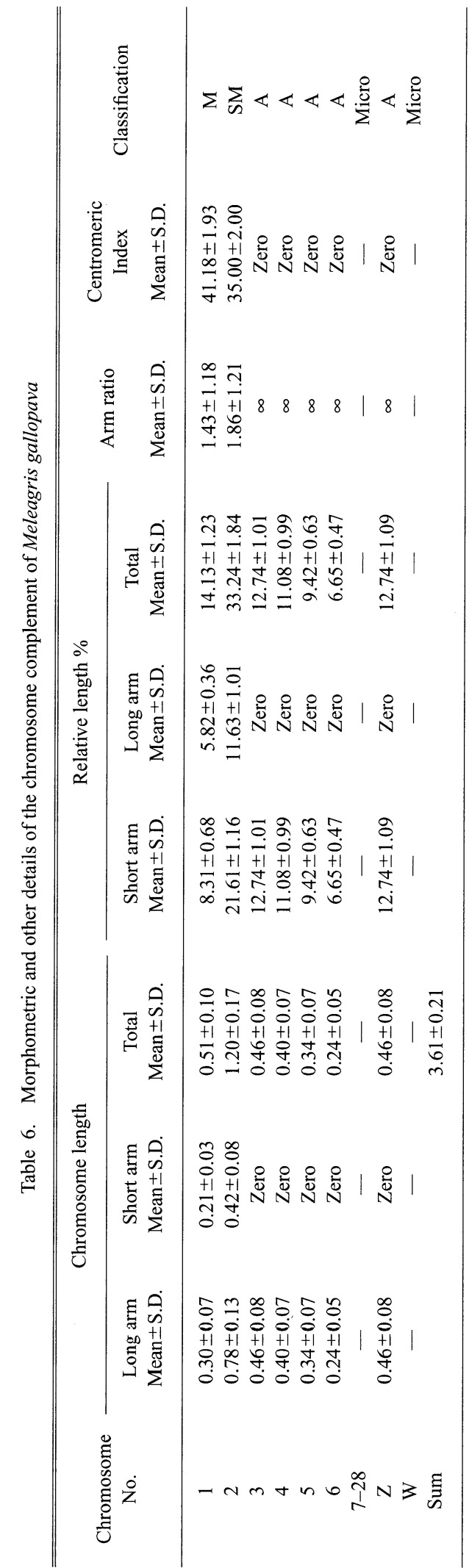




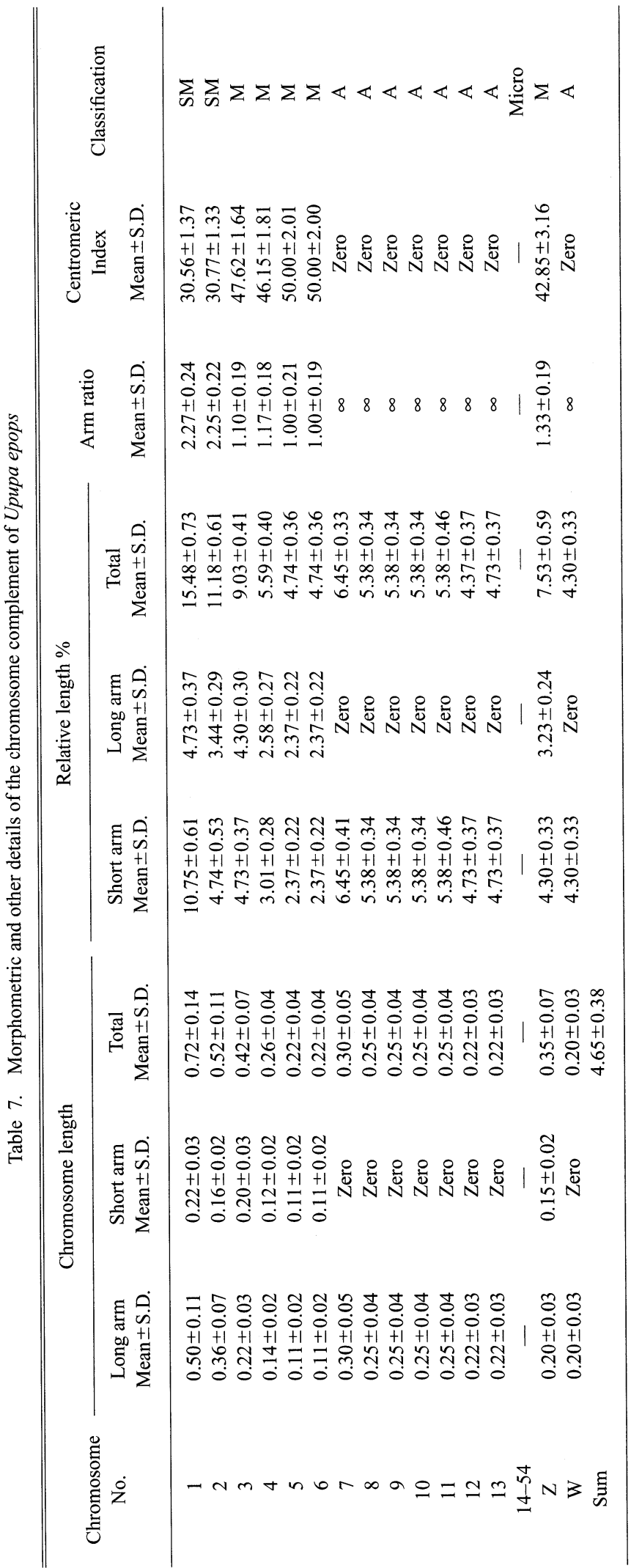




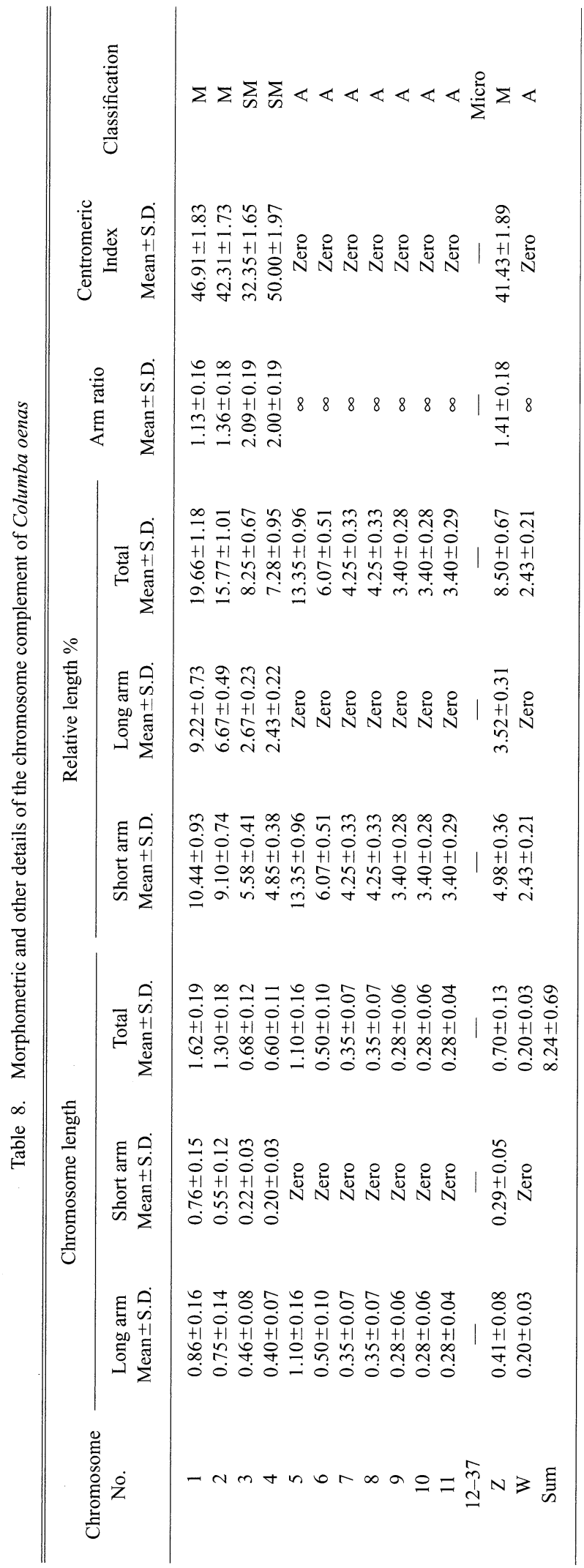




\section{References}

Adegoke, J. A. and Nadesan, S. 1986. Karyotype and polyploidy in the bone marrow of the African fruit bat, Eidolon belvum kerr. Nucleus 29: 107-112.

Benirschke, K. and Hus, T. C. 1971. Chromosome Atlas. Vol. 1, Fish, Amphibia, Reptilia and Birds Spring Verlag, Berlin.

Bhunya, S. P. and Das, K. M. 1991. Karyological study of four Indian birds. Caryologia 44(2): 187-194.

Carlenius, C., Ryttman, H., Tegelstran, H. and Janson, H. 1981. R-, G-, and G-, and G-banded chromosomes in the Domestic Fowl (Gallus domesticus). Hereditas 94: 61-66.

De Boer, L. E. M. 1984. New developments in vertebrate cytotaxonomy. VIII A current references on avian karyology. Genetica 65: 3-37.

Gianfranco, B. 1984. Birds of the World. Macdonal and Co. (Publishers) Ltd., London and Sydney.

Hammar, B. 1970. The karyotype of thirty-one birds. Hereditas 65: 29-58.

Levan, A., Fredga, K. and Sandberg, A. A. 1964. Nomenclature for centromeric position in chromosomes. Hereditas 52: 201-220.

McLaren, A. 1988. Sex determination in mammals. Trends Genet. 4: 153-157.

Mizuno, S. 1991. Evolutionary implication sex chromosome. Specific repetitive DNA sequence in birds belonging to the order, Galliformes. In: New Aspect of the Genetics of Molecular Evolution. Japan Science Society Press, Tokyo/Springer-Verlag, Berlin, pp. 213-226.

Ray-Chaudhuri, R. 1973. Cytotaxonomy and chromosome evolution in birds. In: Cyto-taxonomy and Vertebrate Evolution. Academic Pres, London, New York.

-, Sharama, T. and Ray-Chaudhuri, S. P. 1969. A comparative study of the chromosome of birds. Chromosoma 26: 149-168.

Salama, S. A., Fahmi, A. I. and Abo El-Ghar, G. E. S. 1995. Chromosomal aberration and sperm head abnormalities induced by Abamection and its degradates in male Swiss Albania Mice. Cytologia 60: 411-417.

Sasaki, M., Nishida-Umehara, C. and Tsuchiya, K. 1994. A comparative study of G-banded karyotypes in eight species of Owls. Cytologia 59: 183-185.

Steven, M. G., Peter, M., Sherif, B., Joseph, H. and Wim, M. 1989. The Birds of Egypt. Oxford University Press, New York. Sultana, T. and Bhunya, S. P. 1987. Karyological study of 35 birds from Orissa. Pranikee 8: 65-69.

Tegelstrom, H. and Ryttman, H. 1981. Chromosome in birds (Aves): Evolutionary umplications of macro- and microchromosome numbers and lengths. Hereditas 225-233.

—, Ebebhard, T. and Ryttman, H. 1983. Rate of karyotype and speciation in birds. Hereditas 98-239. 